\title{
The CASSIOPE/e-POP Magnetic Field Instrument (MGF)
}

\author{
D.D. Wallis · D.M. Miles • B.B. Narod • J.R. Bennest • \\ K.R. Murphy $\cdot$ I.R. Mann $\cdot$ A.W. Yau
}

Received: 7 May 2014 / Accepted: 26 September 2014 / Published online: 22 October 2014

(C) The Author(s) 2014. This article is published with open access at Springerlink.com

\begin{abstract}
Field-aligned currents couple energy between the Earth's magnetosphere and ionosphere and are responsible for driving both micro and macro motions of plasma and neutral atoms in both regimes. These currents are believed to be a contributing energy source for ion acceleration in the polar ionosphere and may be detected via measurements of magnetic gradients along the track of a polar orbiting spacecraft, usually the north-south gradients of the east-west field component. The detection of such gradients does not require observatory class measurements of the geomagnetic field. The Magnetic Field instrument (MGF) measures the local magnetic field onboard the Enhanced Polar Outflow Probe (e-POP) satellite by using two ring-core fluxgate sensors to characterize and remove the stray spacecraft field. The fluxgate sensors have their heritage in the MAGSAT design, are double wound for reduced mass and cross-field dependence, and are mounted on a modest $0.9 \mathrm{~m}$ carbon-fiber boom. The MGF samples the magnetic field 160 times per sec $(\sim 50$ meters $)$ to a resolution
\end{abstract}

D.D. Wallis

Magnametrics, Ottawa, Ontario, Canada

D.D. Wallis · A.W. Yau

University of Calgary, Calgary, Alberta, Canada

D.M. Miles $(\varangle) \cdot$ I.R. Mann

University of Alberta, Edmonton, Alberta, Canada

e-mail: David.Miles@ualberta.ca

B.B. Narod

Narod Geophysics Ltd., Vancouver, British Columbia, Canada

B.B. Narod

University of British Columbia, Vancouver, British Columbia, Canada

J.R. Bennest

Bennest Enterprises Ltd., Summerland, British Columbia, Canada

K.R. Murphy

Goddard Space Flight Center, NASA, Greenbelt, MD, USA 
of $0.0625 \mathrm{nT}$ and outputs data at 1952 bytes per second including temperature measurements. Its power consumption is 2.2 watts, and its noise level is $7 \mathrm{pT}$ per root $\mathrm{Hz}$ at $1 \mathrm{~Hz}$.

Keywords Magnetometer $\cdot$ Fluxgate $\cdot$ Satellite $\cdot$ Field-aligned currents

\section{Introduction}

The Enhanced Plasma Outflow Probe (e-POP) is a part of the multi-purpose CASSIOPE small satellite mission sponsored by the Canadian Space Agency (CSA). It is a companion to the Cascade advanced communications technology demonstration payload which serves as a high-speed data downlink for e-POP (as discussed below). The CASSIOPE spacecraft is in an elliptical, polar orbit, with an inclination $80.99^{\circ}$, a perigee of $325 \mathrm{~km}$, and apogee of $1,500 \mathrm{~km}$.

The scientific objective of e-POP is to study plasma outflows, neutral atmospheric escape, and associated effects of auroral currents and plasma microstructures on radio propagation at the highest possible resolution. Knowledge of the magnetic field is essential to this objective (Yau and James 2014). The primary objective of the Magnetic Field Instrument (MGF) on ePOP is the characterization of electric currents flowing into and away from the high latitude (auroral) ionosphere.

Ionospheric currents result in energy being deposited in the ionosphere and drive plasma instabilities which give rise to wave particle interaction and ion acceleration processes (André and Yau 1997) in the topside ionosphere. An integral part of this current system are low-frequency Alfven waves which propagate to the ionosphere from the distant magnetosphere as the magnetic field is being distorted by the convecting plasma. These waves are an important source of both ion and electron acceleration in and above the topside ionosphere (e.g., Watt and Rankin 2009, 2012).

The spatial distribution of the field aligned currents therefore constitutes an essential measurement on e-POP. In the absence of a direct method for measuring currents, the ideal means of determining a field-aligned current distribution is to measure the curl of the magnetic field perturbations resulting from the current and to derive the current distribution based on Maxwell's equations. However, this cannot be done with one spacecraft. Instead we assume the FACs exist as current-sheet structures to reduce the problem to the determination of magnetic field gradients along the spacecraft track. Further, we employ a high cadence fluxgate vector magnetometer to allow us to resolve fine scale current structure. This determination can be successful in the presence of bias fields, such as those that might be present due to spacecraft systems, provided these bias fields are unchanged over the measurement interval.

The bias field from the spacecraft can be estimated and removed from the measured data using a method proposed by Ness et al. (1971). The MGF payload includes two sensors on a single boom at different distances from the spacecraft. The Ness method uses the difference between simultaneous vector measurements taken at different radial distances to estimate the true environmental field and the potentially varying local field generated by the spacecraft. Notably, the Ness method is easiest to apply when the boom is aligned radially with the magnetic center of the spacecraft and is sufficiently long that the dipole term of the spacecraft stray field dominates. Neither of these conditions is well satisfied in the CASSIOPE/e-POP application making the estimation and removal of the spacecraft field more challenging.

Field aligned currents enter and exit the auroral ionosphere with a typical density of $1 \mu \mathrm{A} \mathrm{m}^{-2}$ but on small spatial scales $(\sim 100 \mathrm{~m})$ can exceed $100 \mu \mathrm{Am}^{-2}$; see for example 
Iijima and Potemra (1976) and Rother et al. (2007). The corresponding magnetic gradients are 1.25 and $125 \mathrm{nT}$ per $\mathrm{km}$ or 0.12 and $12 \mathrm{nT}$ per $100 \mathrm{~m}$, respectively. The goal of MGF is the detection of $0.12 \mu \mathrm{A} \mathrm{m}^{-2}$ or stronger currents at scale sizes of $100 \mathrm{~m}$ or larger. This calls for an instrument with resolution of $\sim 0.1 \mathrm{nT}$ and a sample rate of 160 per second.

Elements of the Cascade payload and the CASSIOPE spacecraft bus are intrinsically magnetic, posing difficulties for the measurement of the ambient magnetic field. The MGF sensors are boom mounted to increase the separation of its measurements from the spacecraft's magnetic sources. Two sensors on the boom at two separations are used to estimate and correct for the spacecraft influences.

\section{Instrument Design and Operation}

A fluxgate magnetometer (Primdahl 1979) makes vector measurements of the local magnetic field. It operates by gating the magnetic flux through a solenoid coil and detecting the induced electromagnetic field (EMF). Gating is achieved by periodically driving a specially constructed ring of ferromagnetic material into saturation using an alternating current of frequency $f$ applied with a toroidal winding. The ring is placed in a solenoidal coil. The gated flux, in the presence of an external field, generates an EMF at frequency $2 f$ in the solenoid due to the nonlinear permeability of the ring as it is driven into magnetic saturation (Narod 2014). This second harmonic signal is amplified in an AC amplifier, synchronously detected, integrated, and fed back so as to produce a field opposing and nulling the ambient field in the sensor. The system is carefully designed so that the only means of obtaining a signal at the $2 f$ frequency is the non-linear behavior of the ring core.

The implementation of the over-all fluxgate design for e-POP MGF is illustrated in Fig. 1. The time base circuitry generates a signal at frequency $f=16,457 \mathrm{~Hz}$ which is applied to the ring, driving it into saturation twice per cycle. The EMF induced by the changing flux in the sensor winding passes through a $2 f$ band-pass filter and is synchronously detected. Parametric gain is achieved by capacitively loading the sensor (Narod and Russell 1984). The demodulated $2 f$ signal is then integrated and digitized, and fed back through a trans-conductance amplifier to the sense/feedback winding. Each component incorporates a digital to analog converter (DAC) to bias the operating range of the instrument. This biasing technique is not commonly used but has been successfully employed by the CARISMA (previously CANOPUS) magnetometers (Mann et al. 2008; Rostoker et al. 1995) used on the Earth's surface.

The design of the MGF instrument was motivated by the measurement objectives in terms of sensitivity and sampling rate. A ranging magnetometer design was chosen as a suitable digitizer capable of the range of expected field values $( \pm 65,536 \mathrm{nT})$ was not available at the desired resolution $(0.0625 \mathrm{nT})$ and sampling rate (160 samples per second) at the beginning of the e-POP project. A 12 bit analog to digital converter (ADC) digitizes the analog magnetometer signal in the range of -128 to $+127 \mathrm{nT}$. A 17 bit DAC (16 bit integrated circuit with an external analog switch providing polarity control) with a $1 \mathrm{nT}$ least significant bit applies an offset current to the combined sense/feedback winding to set the range under digitization. The output of the instrument is a combination of the DAC input bits and the ADC output bits. Each channel is restricted to steps of 64,96 , or $128 \mathrm{nT}$ to reduce telemetry requirements and the frequency of offset adjustments. The bottom 5 bits of the DAC are therefore zero and are not telemetered.

The chosen DAC has integral and differential non-linearity of 0.2 least significant bit (LSB). Offset changes inevitably introduce a step function in the analog magnetometer cir- 


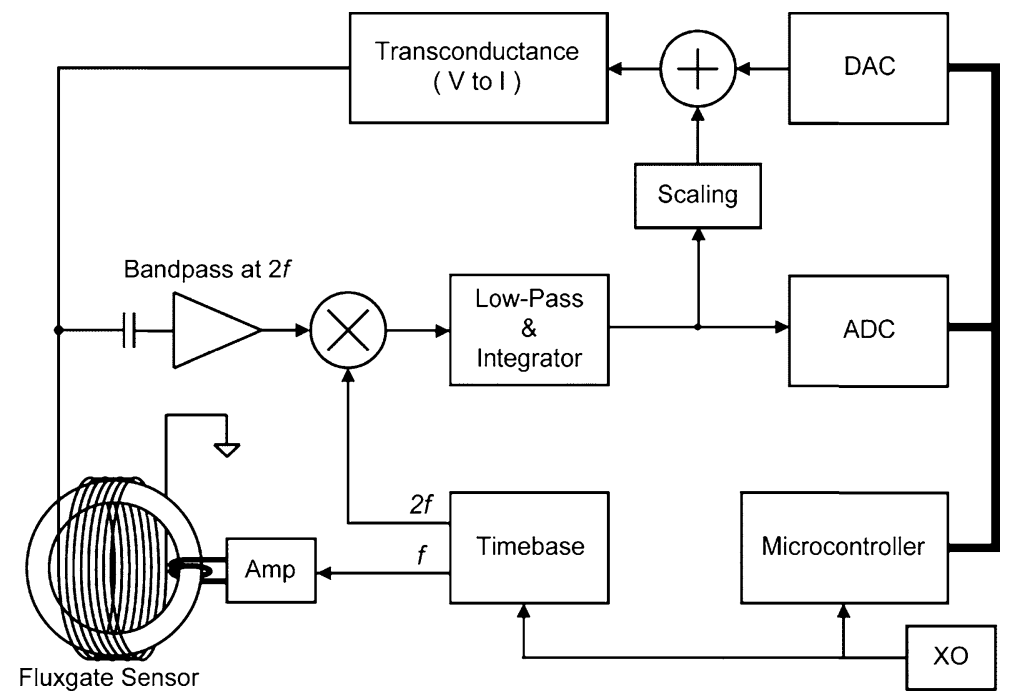

Fig. 1 Simplified diagram of the MGF instrument showing the major functional blocks in each magnetometer

cuit which will introduce a transient in the combined digital output. Offset changes are synchronous with the time base governing the sampling and are allowed only every 4th sample so they can be removed in post-processing.

The sensor design has its heritage in the NASA MAGSAT fluxgate design (Acuña et al. 1978). The S1000 ring cores were manufactured by Infinetics. They are close wound toroidally and cemented in their Macor feedback coil bobbins with room temperature vulcanizing (RTV) silicone. The combined feedback/sense coils are 360 turns of insulated copper wire wound under tension. The thermal expansion of Macor is close to that of the rings and the tensioned winding effectively makes the thermal expansion of the copper wire equal to that of the Macor. Two orthogonal solenoidal coils are wound on each bobbin, providing two measurements in the plane of each ring. Figure 2 shows the two double-wound coil assemblies orthogonally mounted on a Macor block which is machined to mount on the magnetometer boom.

The X-Y, X-Z, and Y-Z angles between the sensitive axes have similar tolerances to a standard three ring sensor and were measured to be $89.9347^{\circ}, 90.0239^{\circ}$, and $89.9326^{\circ}$ respectively for the outer sensor and $90.1153^{\circ}, 90.1047^{\circ}$, and $89.8066^{\circ}$ respectively for the inner sensor. Our adoption of a two ring design resulted in a significant reduction in mass (as only two rather than three rings and sensor bobbins are needed) and in the reduction of the cross field sensitivity to near zero. Cross field sensitivity is an undesirable response to the orthogonal field component in the plane of the ring (Acuña et al. 1978). By simultaneously zeroing both components, cross field sensitivity is minimized.

In this design, one ring gates the flux in the $\mathrm{X}$ and $\mathrm{Z}$ directions while the second ring gates the $\mathrm{Y}$ and $\mathrm{Z}$ directions; $\mathrm{X}$ and $\mathrm{Z}$ being the nominal spacecraft ram face (out of the page in Fig. 6 below) and nominal nadir direction (down in Fig. 6 below), respectively. Figure 3 shows how the $\mathrm{Z}$ feedback coils are wired in series, so that the final result for the $\mathrm{Z}$ component is the average of the $\mathrm{Z}$ fields at the two rings.

The MGF incorporates two independent and almost identical electronics boards. Each board contains its own DC-to-DC switched power converter, to allow each one to operate 
Fig. 2 Each MGF sensor is constructed from two orthogonal ring-cores, each with a combined sense/feedback winding
Fig. 3 Wiring diagram for the fluxgate sensor. Channels $\mathrm{X}$ and $\mathrm{Y}$ are each derived from orthogonal sense/feedback windings. Channel $\mathrm{Z}$ is derived from two sense/feedback windings connected in series. The two rings and their respective windings are mounted orthogonally as shown in the inset
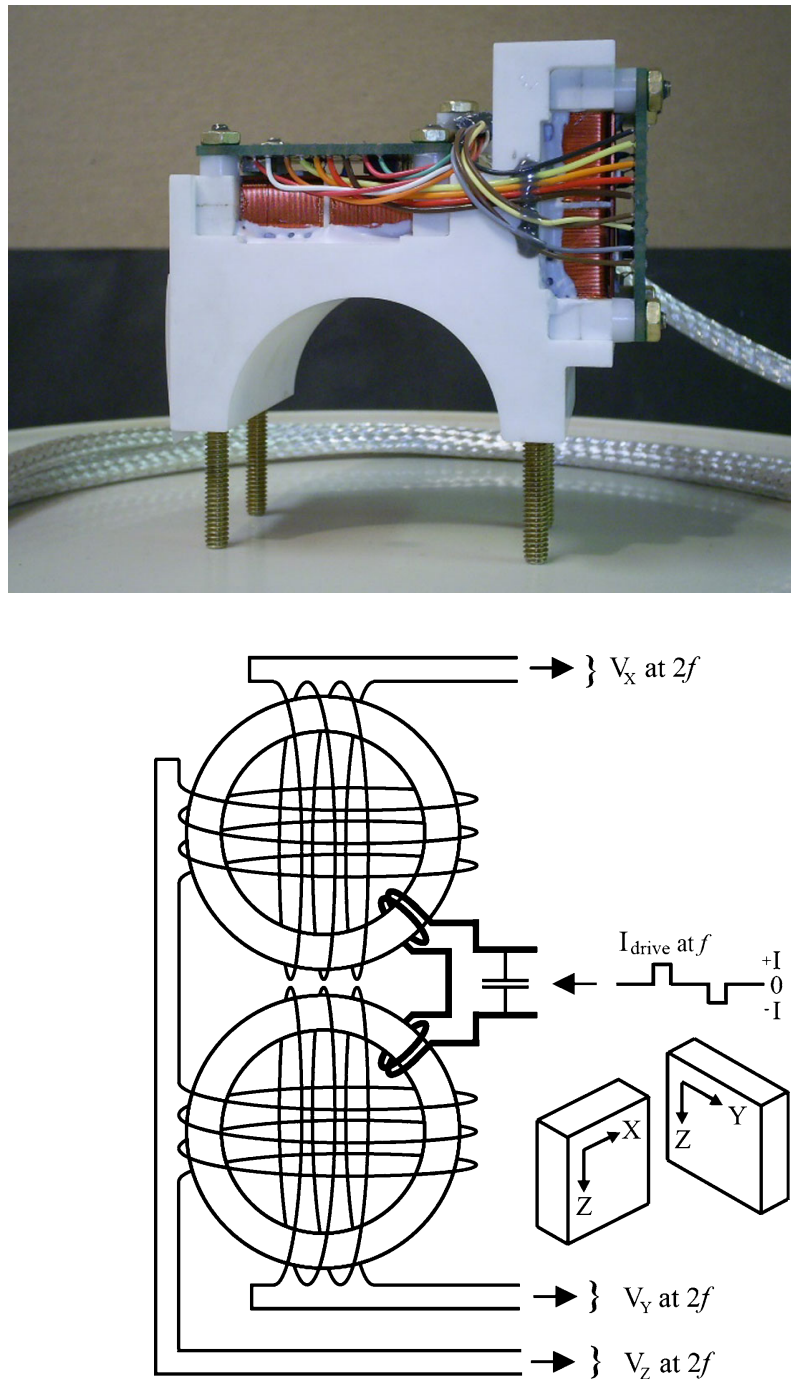

independently should the other fail. Both boards share the data, command and power interfaces. Figure 4 shows the MGF flight electronics box and connectors. The top and bottom ports are for the two fluxgate sensors. The two middle ports are for power and communication with the spacecraft bus, respectively.

Each board has its own crystal oscillator, excitation drive circuitry, controller, and magnetometer circuitry with DACs and ADCs for each measured component. The crystal oscillator on each board is cross-strapped with the other board to serve as backup for the other oscillator in the case of failure. At power up, a failure tolerant voting circuit selects one oscillator to drive both boards synchronizing the two magnetometers. One power feed is shared by the two cards and each provides data on a RS-422 synchronous interface to the e-POP Data Handling Unit (DHU). Time multiplexing of the data from each magnetometer allows sharing of the interface to the DHU. Data transmissions is synchronized to the spacecraft-generated $1 \mathrm{~Hz}$ clock. Additionally the cards are wired so that if the $1 \mathrm{~Hz}$ space- 
Fig. 4 MGF flight electronics box, with the top and bottom covers removed. The top and bottom ports are for the two fluxgate sensors. The two middle ports are for power and communication with the spacecraft bus
Fig. 5 Electronics card for one of the MGF magnetometers, with the electronic components staked for flight and an external programmer connected for testing and reloading of the onboard microcontroller
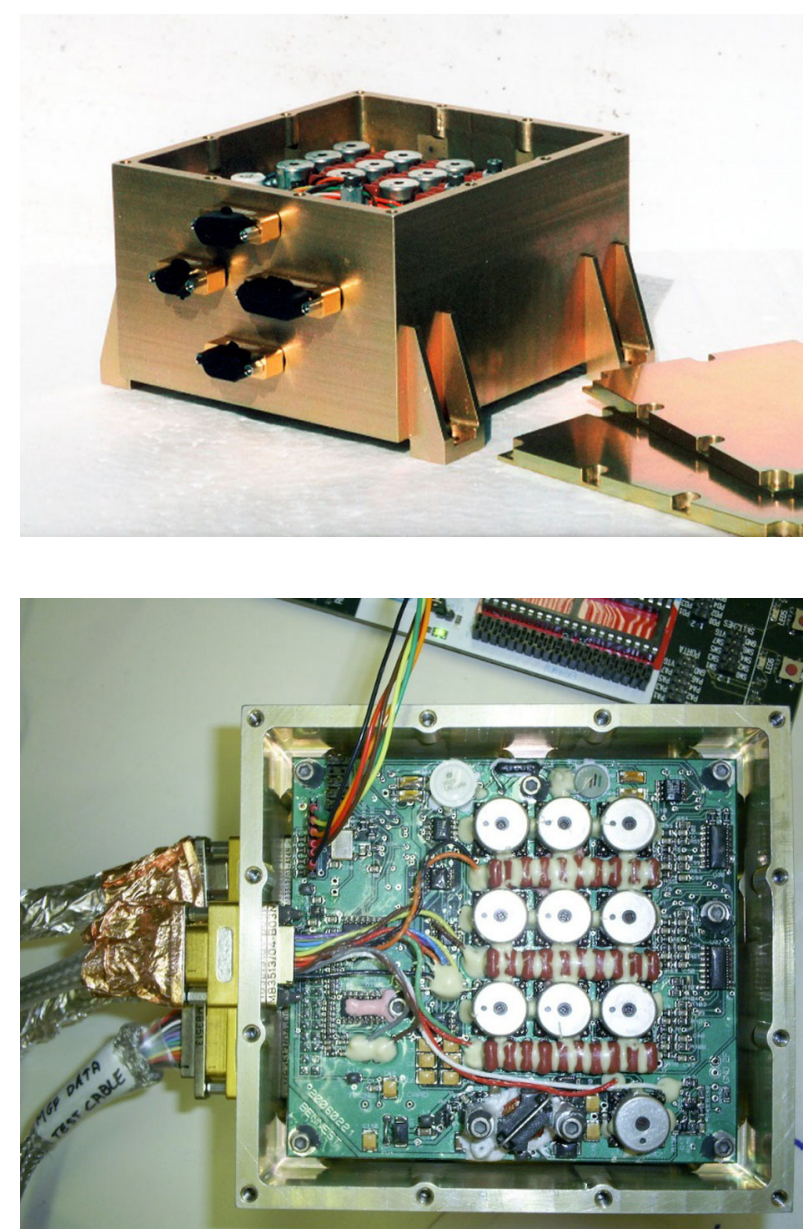

craft clock is unavailable for synchronization at power-up, the card currently in "Master" mode continues to operate while the other card in "Slave" mode goes dormant and will not transmit.

The magnetometer data comprise 160 12-bit ADC samples per second for each of three magnetic components. The DAC for each component is updated and telemetered as a 12-bit value 40 times per second (every fourth ADC value). The data require 900 bytes per second per magnetometer. Two data blocks are transmitted to the data handling unit per second, one for each of the magnetometers. Each data block is 976 bytes long and contains 76 bytes of housekeeping and status data, memory checksums and software flags.

The employed controller chip is the Atmel ATMEGA32L 8-bit microprocesssor, clocked at $3.6864 \mathrm{MHz}$. Its on-chip digitizers monitor the temperatures of both sensors and both electronics boards, and voltages derived from the internal voltage rails. Figure 5 shows the electronics card for one of the magnetometers staked and mounted for flight.

The MGF is intended to operate continuously whenever possible, to maximize the data acquisition both inside and outside the auroral zone and the polar cap. The MGF data samples are time-stamped against the spacecraft $1 \mathrm{~Hz}$ pulse, which is derived from the onboard GPS receivers. A custom firmware bootloader allows the MGF firmware to be reloaded dur- 
Fig. 6 MGF in deployed configuration showing the inboard and outboard fluxgate sensors

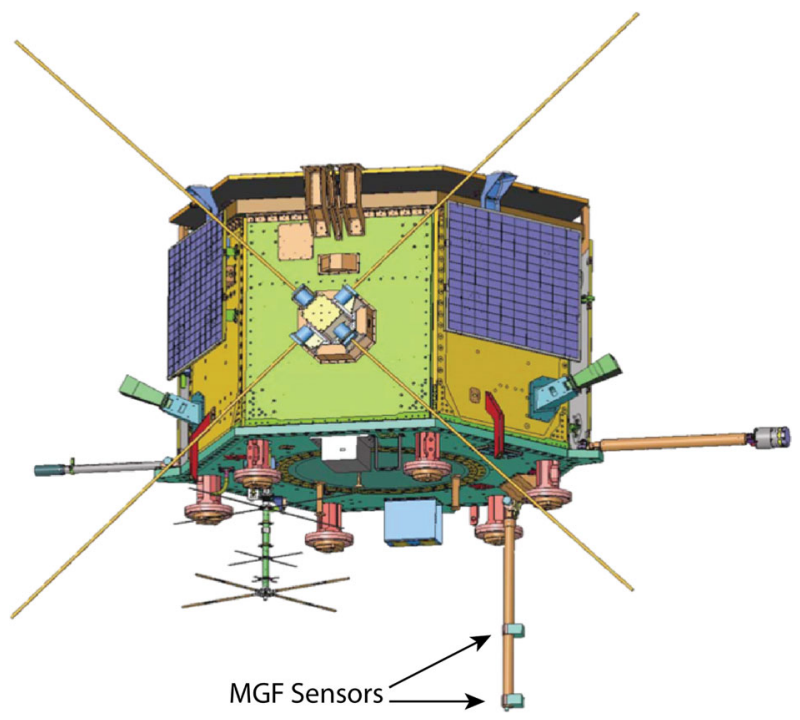

ing flight via spacecraft command. Essentially any subset or the full set of the firmware may be reloaded.

As the temperature of the sensors varies, the physical dimensions of the feedback coil change. Nulling a given field component then requires a different current from the trans-conductance amplifier. Additionally, the resistance of the copper wire making up the feedback coil changes, producing another source of temperature variation. The transconductance amplifier senses the voltage of the feedback current circuit which is fed back to the input via a temperature compensation network (Acuña et al. 1978). The load resistance (and its temperature dependence) is dominated by a $100 \mathrm{ohm}$ platinum resistor in series with the feedback coil. This platinum resistor is cemented to the feedback winding with Nusil CV2942 thermally conductive elastomer. Changes in sensor temperature cause a linear change in gain scaling which is measured to be $-0.6,-1.3$, and $+1.2 \mathrm{nT} /{ }^{\circ} \mathrm{C}$ in the $\mathrm{X}, \mathrm{Y}$, and $\mathrm{Z}$ channels respectively. Sensor temperature has no measurable effect on the instrumental zeroes.

A carbon fiber boom is used to position the sensors at a modest distance from the spacecraft. At launch the boom was in a stowed configuration, folded at the base of the CASSIOPE spacecraft and held in place by a hinge mechanism at one end and a deployment actuator and a pin puller arrangement at the other. The deployment actuator provides a positive deployment force when a small pin is removed from a plug in the end of the boom. The pin puller is of the melting wax type employing a redundant heater element. The hinge assembly utilizes a viscous damper to slow the deployment and a locking wedge to secure the deployed boom. A potentiometer was used to measure the hinge angle. The MGF boom was released early in the commissioning phase of the mission and deployed to an angle of $89.2^{\circ} \pm 1^{\circ}$. Figure 6 depicts the MGF in the deployed configuration, showing the inboard and outboard fluxgate sensors.

Carbon fiber was chosen to eliminate fields due to eddy currents that would be induced in a metallic boom. Such fields were estimated to be $\sim 10 \mathrm{nT}$ at times of large changes of the ambient field. The boom was machined to achieve a smooth surface at the mounting locations of the sensor assembly. The bases of the sensors were machined to have a semi-circular saddle of the same diameter as the machined boom. A thin layer of thermally 
conductive RTV silicone was applied to the boom before final assembly. A mounting bracket made of aluminum was cemented to the back side of the boom to provide a reference surface for remounts during tests and calibrations. The entire boom was covered with a multilayer conductive blanket for thermal control.

Since the thermal conductivity of Macor is moderately high, the thermal coupling between the Macor bases and the boom provides a large thermal mass and reduces the temporal changes of the temperature of the sensors (which has generally been in the range of -15 to $-30{ }^{\circ} \mathrm{C}$ on orbit). A small, 2-watt survival heater situated midway between the two sensors on the boom is programmed to activate at $-40{ }^{\circ} \mathrm{C}$ and deactivate at $-38{ }^{\circ} \mathrm{C}$. The controlling thermistor is mounted at the outboard sensor bracket. The current required by the survival heater should create a detectable stray magnetic field at both sensors. However, there was no opportunity to characterize this before launch and, since commissioning, there has never been an MGF observing session where the sensor was sufficiently cold to activate the heater.

The CASSIOPE/e-POP satellite can control its attitude using either reaction wheels or magnetic torque rods. Both techniques impact the magnetic field measurement. However, the torque rods render the magnetic data unusable so the spacecraft is normally restricted to reaction wheels during MGF observing sessions. The instrument is saturated (but is not damaged) by exposure to the fields from the torque rods of approximately 1,000 to $2,000 \mathrm{nT}$ at the boom end. The instruments servo rapidly back into the range of the environmental field after the torque rods finish firing. The four reaction wheels are normally idle with a frequency near $15 \mathrm{~Hz}$ but vary between 10 and $20 \mathrm{~Hz}$ during maneuvers. The combined magnetic signature of the four wheels is a varying envelope sinusoid with a peak to peak amplitude of 10-20 nT. Low-pass filtering the measured data removes the majority of the reaction wheel tones. More sophisticated filtering is in development to suppress the reaction wheel interference in the full cadence data.

The stray spacecraft field of the CASSIOPE/e-POP satellite can be estimated and removed using the dual-magnetometer technique described by Ness et al. (1971). It was not possible to fully characterize the stray magnetic fields of the spacecraft in its many different operational configurations before launch. The stray field is therefore estimated from in-flight data by treating it as a dipole field at the center of the spacecraft. The estimation is further simplified by assuming that the two magnetometers are co-aligned and radial to the spacecraft dipole. The measurement from each sensor is corrected using an orthogonality matrix and then rotated into the frame of the boom before the dual magnetometer technique is applied. These assumptions allow us to create a dynamic estimate of the spacecraft field which can be subtracted from the measured field to obtain the corrected estimate of the ambient magnetic field. It should be noted that it is not possible to separate instrumental zero drift and spacecraft fields solely from on-orbit measurements. However, the zeroes can be independently estimated using spacecraft roll maneuvers. The dual magnetometer method was illustrated by Acuña (2002) and was employed recently in the Double Star mission (Carr et al. 2005).

The relative proximity of the two sensors introduces crosstalk between them. The separation is governed by the length of the boom and the magnitude of the spacecraft field components at the sensors. Each sensors acts to null the field in its solenoidal coils which generates a stray surrounding magnetic field. In testing, the perturbation of a 50,000 nT field on the $\mathrm{X}, \mathrm{Y}$, and $\mathrm{Z}$ components by the other sensor is 2,2 and $13 \mathrm{nT}$, respectively. The effect is linear with ambient field. The $\mathrm{Z}$ component is larger because it comprises two coils per magnetometer (increasing the size of the source) and the $\mathrm{Z}$ axis is parallel to the boom while 


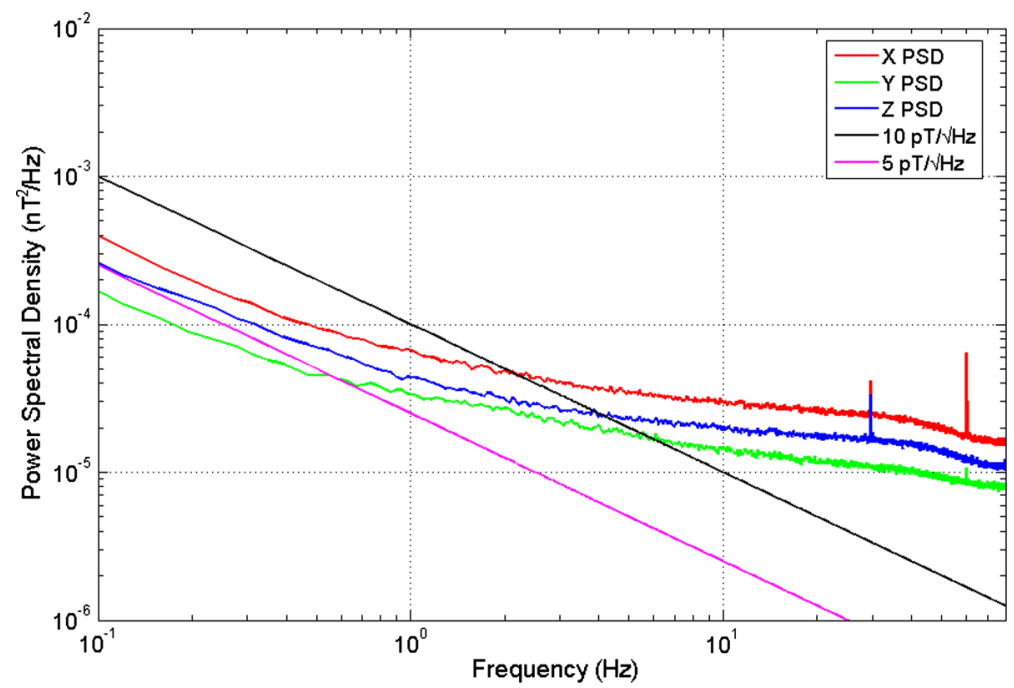

Fig. 7 Engineering model magnetometer frequency spectra

Table 1 Spacecraft resources required by the MGF

\begin{tabular}{llll}
\hline MGF component & Mass $(\mathrm{kg})$ & Power (watts) & Dimensions (mm) \\
\hline Electronics box & 1.22 & 2.2 & $130 \times 110 \times 70$ \\
Sensors (2) & 0.58 & 0.2 & $70 \times 50 \times 60$ \\
Sensor harness & 0.35 & - & - \\
Boom & 1.17 & - & $44 \times 900$ \\
Boom hardware & 1.11 & - & - \\
\hline
\end{tabular}

Table 2 Key science measurement parameters achieved by MGF

\begin{tabular}{ll}
\hline Data rate & $1.9 \mathrm{kbps}$ \\
Sampling cadence & $160 \mathrm{sps}$ \\
Magnetic range & $\pm 65,536 \mathrm{nT}$ \\
Resolution & $0.0625 \mathrm{nT}$ \\
\hline
\end{tabular}

$\mathrm{X}$ and $\mathrm{Y}$ axes are orthogonal. This interaction is scaled and removed from the flight data in post-processing.

Table 1 lists the spacecraft resources required by MGF and Table 2 summarizes the key science measurement parameters described in this section.

Before spacecraft integration, the two e-POP MGF flight magnetometers were calibrated individually at the Geomagnetic Laboratory of National Resources Canada near Ottawa. Sensitivity and directions of the sensitive axes with respect to the boom were determined, as well as the temperature dependence of both the electronics and the sensors. The magnetometers were found to exhibit no meaningful sensitivity to the supply voltage. The cross-talk between the boom mounted sensors was also characterized.

Figure 7 presents power spectral density measurements acquired from the engineering model electronics card and the Infinetics core-based sensor. These data are comparable to 
the performance achieved by both flight instruments. These spectra are taken with the sensor operating in a 6 layer magnetic shield at effectively zero field at all frequencies. Two narrow features are present at 30 and $60 \mathrm{~Hz}$. These are likely residuals of the AC power lines in the laboratory. The spectra show the expected $1 / f$ frequency dependence of the noise floor, which is below $10 \mathrm{pT}$ per $\mathrm{Hz}^{1 / 2}$ at $1 \mathrm{~Hz}$ and achieves our goal of resolving currents of $0.1 \mu \mathrm{A} \mathrm{m}^{-2}$ in 100 - $\mathrm{m}$ wide structures.

\section{MGF Data Products}

Several data products are created from the MGF measurements. Level 0 and 1 data are not expected to the useful to the general science user and are therefore not routinely distributed. In addition, MGF generates Level 2 and 3 data on a routine basis. Level 2 data reconstructs the fully calibrated magnetic measurement from each sensor rotated into the reference frame of the spacecraft, and is useful for the analysis of the particle distribution measurements taken by other science instruments. Level 3 data use the Ness et al. (1971) dual magnetometer technique described above to estimate and remove the spacecraft magnetic field. Level 3 data contains single three-component estimates of the local field. Resampled perfectly second-aligned 10-samples per second (sps) and 1-sps time series are also produced. These time series are available in the spacecraft coordinate system and rotated into the Geocentric Equatorial Inertial (GEI) coordinate system. Quick look and summary plots are created from Level 2 data for each MGF orbit pass. The various MGF data products including the data plots are available online through the Canadian Space Science Data portal (http://www.cssdp.ca).

\section{Example of MGF Observation Data}

Figure 8a shows ground tracks for two consecutive conjunctions over the Fort Smith all sky imager (Mende et al. 2009) crossing the North American sector of the night side auroral oval from between 56 and 65 degrees latitude on February 27 and 28, 2014. Both orbits pass near the center of the field of view of the Fort Smith all sky imager (ASI) at 09:18:36 UT. During these passes the e-POP Fast Auroral Imager (FAI) payload was not operating hence we use THEMIS ground based ASI data. The spacecraft was in Nadir pointing mode so, in the spacecraft coordinate system, $+\mathrm{Z}$ is in the Nadir (down) direction, $+\mathrm{X}$ is in the direction of spacecraft motion (approximately northward), and $+Y$ completes the right-handed coordinate system (approximately eastward). Field aligned currents can be detected as perturbations in the local magnetic field compared to the nominal background. In this analysis, the cross-track spacecraft $\mathrm{Y}$ component was selected to minimize the effect of orbit and attitude uncertainty as discussed by Anderson et al. (2000). February 27 was a geomagnetically quiet day with no significant auroral activity in the region of interests (Fig. 8b). The pass on February 28 intersected several auroral forms between 56 and 65 degrees latitude (Fig. 8c) during a substorm expansion phase which initiated at 07:51 UT.

Figure 9a shows the magnetic field measurement in the spacecraft Y component (crosstrack, approximately eastward) versus latitude on February 27 and 28 as respectively red and blue. The magnetic data have been low-pass filtered at $4.5 \mathrm{~Hz}$ to suppress the stray magnetic field from the four reaction wheels used to steer the spacecraft. Figure $9 \mathrm{~b}$ compares the FAC densities observed during each pass. The FAC densities are approximated using the spacecraft $\mathrm{Y}$ component magnetic field and $\mathrm{X}$ component velocity of the spacecraft as 

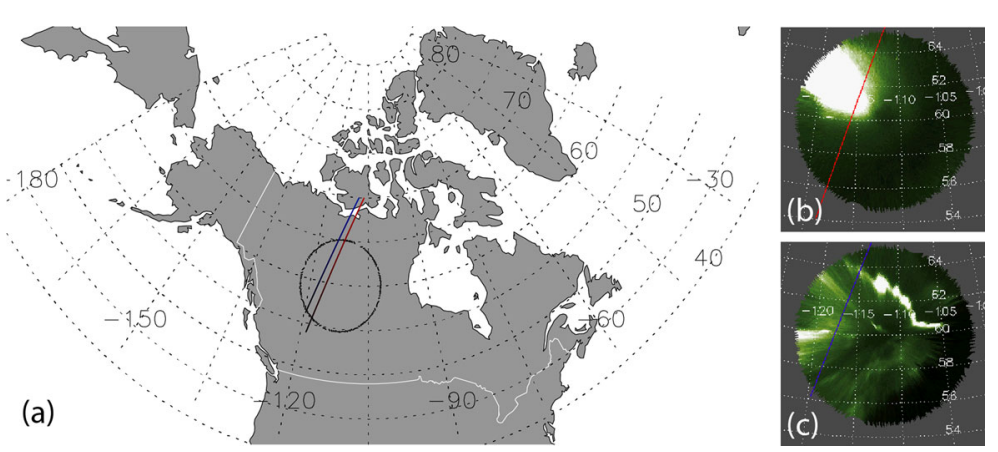

Fig. 8 (a) Field of view of the Fort Smith all sky imager and spacecraft track for February 27 (black to red shows poleward spacecraft motion) and 28 (black to blue shows poleward spacecraft motion). (b) Fort Smith all sky camera February 27, 2014 at 09:18:36 UT with superposed spacecraft track in red. (c) Forth Smith all sky camera February 28, 2014 at 09:18:36 UT with superposed spacecraft track in blue

described in Potemra (1985). This approximation ignores perturbations in the spacecraft X magnetic field component and assumes that the spacecraft motion is predominantly along the spacecraft X direction. Positive (negative) denotes upward (downward) FACs or downward (upward) going electrons. It is clear from Fig. 9b that the FAC amplitudes are both stronger and more dynamic in the presence of the aurora.

The relation between auroral features and FAC intensities is better illustrated by comparing the along-track auroral intensity from the Fort Smith ASI with the estimated FACs. This is shown in Figs. 9c and 9d. Figure 9c plots the FAC from February 28 (same as Fig. 9b) with the along-track auroral intensity. The FAC strength is shown in blue and the auroral intensity in green. Figure 9d plots the average FAC intensity over a spatial scale of 0.25 degrees $(\sim 60 \mathrm{~km})$, the approximate scale size of auroral features observed in the Fort Smith ASI by smoothing the data at 4 seconds with the along track auroral intensity (in the same format as Fig. 9c). During the auroral pass it is clear that FACs are both large and very dynamic with the largest FAC strengths observed at the peak in auroral intensity at $\sim 60$ degrees latitude. Averaging over 0.25 degrees $(\sim 60 \mathrm{~km})$, Fig. $9 \mathrm{~d}$, shows a net upward FAC in the region between 59.5-61.5 degrees. This matches the peak in auroral intensity where we would expect to find downward going electrons. Similarly, between $\sim 58.5-59.5$ degrees a secondary peak in auroral intensity is associated with a net upward FAC. Conversely the peak in auroral intensity between 61.5-62 degrees is associated with a net downward FAC. This is likely an artifact of using a single magnetic field component in spacecraft coordinates and a simple approximation to calculate FACs.

In general, the magnetic signature of field aligned currents is more accurately determined from the deviation from the theoretical International Geomagnetic Reference Field (IGRF) (e.g., Anderson et al. 2000 and Waters et al. 2001). This requires accurate background field removal, which at this time is limited by the processing of the spacecraft attitude solution which introduces artifacts into the subtraction of the background field from the measured spacecraft field. Once these issues are resolved we intend to routinely generate and distribute FACs estimated from the east-west and north-south magnetic field perturbations to provide a more robust FAC estimate. We also intend to integrate the e-POP magnetic field data into the AMPERE and Iridium determinations of FACs on a case-by-case basis to provide global estimates of FACs from multiple space craft. 
Fig. 9 (a) Spacecraft Y magnetic field (eastward) versus latitude from February 27 quiet day (red) and February 28 as the spacecraft passes through an active auroral region (blue).

(b) FAC amplitudes for February 27 (red) and February 28 (blue). (c) FAC amplitude on February 28 (blue) and along-track auroral intensity from the Fort Smith all sky imager (green). (d) FAC amplitudes on February 28 (blue) averaged at 4 seconds to show the net FAC density on the spatial scale of the auroral forms observed at the Fort Smith all sky imager. This smoothing corresponds to approximately $60 \mathrm{~km}$ scales, or 0.5 degrees in latitude. The along-track intensity from the Fort Smith all sky imager is shown in green. In panels (b)-(d) positive denotes upward FACs or downward going electrons
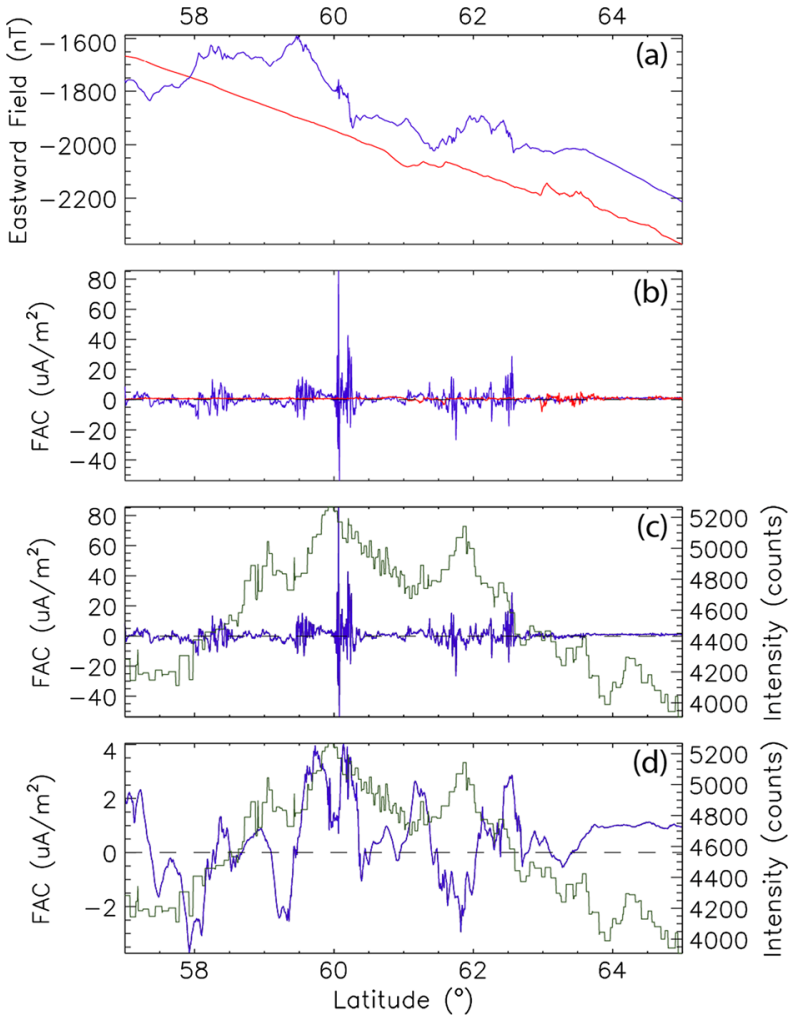

\section{Conclusions}

Field-aligned currents may be detected via measurements of magnetic gradients along the track of a polar orbiting spacecraft (usually the north-south gradients of the east-west field component). The Magnetic Field Instrument (MGF) on the Enhanced Plasma Outflow Probe (e-POP) measures the local magnetic field onboard by using two ring-core fluxgate sensors to characterize and remove the stray spacecraft field. The fluxgate sensors draw their heritage from the MAGSAT design, and the instrument relies on a ranging design that was previously adopted successfully in the CARISMA ground-based magnetometers. Sensor mass has been reduced by using each ring core to sense two components of the field. This has the added benefit of minimizing cross-field dependence. The MGF samples the magnetic field at 160 samples per sec $(\sim 50$ meters $)$ to a resolution of $0.0625 \mathrm{nT}$ and has a noise level of $7 \mathrm{pT}$ per root $\mathrm{Hz}$ at $1 \mathrm{~Hz}$. Data in the first few months of the mission has demonstrated the potential of the instrument to achieve its scientific goal of providing high fidelity measurements of field aligned current structures in the high-latitude auroral ionosphere.

Acknowledgements We acknowledge the support from the Canadian Space Agency for the development and operation of the CASSIOPE/e-POP mission. The authors are grateful for the support and guidance of R. Hum. We thank J. Schmidt of Minerva Technology Inc. for his contributions to the flight firmware, W. Lunscher and his team at COM DEV for their technical assistance, and the technical team at Magellan Aerospace Corporation for the boom development. K.R. Murphy is supported by an NSERC Postdoctoral fellowship. D.M. Miles is supported by grants from the Canadian Space Agency and a Canadian NSERC PGSD2 graduate scholarship. I.R. Mann is supported by an NSERC Discovery Grant. A.W. Yau is supported by grants from the Canadian Space Agency and the NSERC Industrial Research Chair and Discovery Grant programs. 
Open Access This article is distributed under the terms of the Creative Commons Attribution License which permits any use, distribution, and reproduction in any medium, provided the original author(s) and the source are credited.

\section{References}

M.H. Acuña, Space based magnetometers. Rev. Sci. Instrum. 73, 3717-3736 (2002). doi:10.1063/1.1510570

M.H. Acuña, S.C. Scearce, J.B. Seek, J. Scheifele, The MAGSAT Vector Magnetometer-a precision fluxgate magnetometer for the measurement of the Geomagnetic field. NASA Technical Memorandum, 79656 (1978)

B.J. Anderson, K. Takahashi, B.A. Toth, Sensing global Birkeland currents with Iridium ${ }^{\circledR}$ engineering magnetometer data. Geophys. Res. Lett. 27(24), 4045-4048 (2000). doi:10.1029/2000GL000094

M. André, A.W. Yau, Theories and observations of ion energization and outflow in the high latitude magnetosphere. Space Sci. Rev. 80(1-2), 27-48 (1997). doi:10.1023/A:1004921619885

C. Carr, P. Brown, T.L. Zhang, J. Gloag, T. Horbury, E. Lucek, W. Magnes, H. O’Brien, T. Oddy, U. Auster, P. Austin, O. Aydogar, A. Balogh, W. Baumjohann, T. Beek, H. Eichelberger, K.-H. Fornacon, E. Georgescu, K.-H. Glassmeier, M. Ludlam, R. Nakamura, I. Richter, The Double Star Magnetic Field Investigation: instrument design, performance and highlights of the first year's observations. Ann. Geophys. 23(8), 2713-2732 (2005). <hal-00317923>

T. Iijima, T.A. Potemra, The amplitude distribution of field-aligned currents at northern high latitudes observed by Triad. J. Geophys. Res. 81(13), 2165-2174 (1976). doi:10.1029/JA081i013p02165

I.R. Mann, D.K. Milling, I.J. Rae, L.G. Ozeke, A. Kale, Z.C. Kale, K.R. Murphy, A. Parent, M. Usanova, D.M. Pahud, E.-A. Lee, V. Amalraj, D.D. Wallis, V. Angelopoulos, K.-H. Glassmeier, C.T. Russell, H.U. Auster, H.J. Singer, The upgraded CARISMA magnetometer array in the THEMIS era. Space Sci. Rev. 141(1-4), 413-451 (2008). doi:10.1007/s11214-008-9457-6

S.B. Mende, S.E. Harris, H.U. Frey, V. Angelopoulos, C.T. Russell, E. Donovan, B. Jackel, M. Greffen, L.M. Peticolas, The THEMIS array of ground-based observatories for the study of auroral substorms, in The THEMIS Mission (Springer, New York, 2009), pp. 357-387

B.B. Narod, The origin of noise and magnetic hysteresis in crystalline permalloy ring-core fluxgate sensors. Geosci. Instrum. Methods Data Syst. 4, 319-352 (2014). doi:10.5194/gi-3-201-2014

B.B. Narod, R.D. Russell, Steady-state characteristics of capacitively loaded fluxgate sensor. IEEE Trans. Magn. 20, 592-597 (1984). doi:10.1109/TMAG.1984.1063122

N.F. Ness, K.W. Behannon, R.P. Lepping, K.H. Schatten, Use of two magnetometers for magnetic field measurements on a spacecraft. J. Geophys. Res. 76, 3565-3573 (1971). doi:10.1029/JA076i016p03564

T.A. Potemra, Field-aligned (Birkeland) currents. Space Sci. Rev. 42, 295-311 (1985). doi:10.1007/ BF00214990

F. Primdahl, The fluxgate magnetometer. J. Phys. E, Sci. Instrum. 12, 241-253 (1979). doi:10.1088/0022$3735 / 12 / 4 / 001$

G. Rostoker, J.C. Samson, F. Creutzberg, T.J. Hughes, D.R. McDiarmid, A.G. McNamara, A. Vallance Jones, D.D. Wallis, L.L. Cogger, CANOPUS - a ground-based instrument array for remote sensing the high latitude ionosphere during the ISTP/GGS program. Space Sci. Rev. 71(1-4), 743-760 (1995). doi:10.1007/BF00751349

M. Rother, K. Schlegel, H. Lühr, CHAMP observation of intense kilometer-scale field-aligned currents, evidence for an ionospheric Alfvén resonator. Ann. Geophys. 25(7), 1603-1615 (2007). doi:10.5194/ angeo-25-1603-2007

C.L. Waters, B.J. Anderson, K. Liou, Estimation of global field aligned currents using the Iridium ${ }^{\circledR}$ system magnetometer data. Geophys. Res. Lett. 28(11), 2165-2168 (2001). doi:10.1029/2000GL012725

C.E.J. Watt, R. Rankin, Electron trapping in shear Alfvén waves that power the aurora. Phys. Rev. Lett. 102, 045002 (2009). doi:10.1103/PhysRevLett.102.045002

C.E.J. Watt, R. Rankin, Alfven wave acceleration of auroral electrons in warm magnetospheric plasma, in Auroral Phenomenology and Magnetospheric Processes: Earth and Other Planets, ed. by A. Keiling, E.F. Donovan, F. Bagenal, T. Karlsson. Geophysical Monograph Series (American Geophysical Union, Washington D.C., 2012), p. 251

A.W. Yau, H.G. James, The CASSIOPE Enhanced Polar Outflow Probe (e-POP) mission overview. Space Sci. Rev. (2014 this issue) 